\title{
Orthogonal decomposition of the sum-symmetry model using the two-parameters sum-symmetry model for ordinal square contingency tables
}

\author{
Shuji Ando \\ Department of Information and Computer Technology, Faculty of Engineering, \\ Tokyo University of Science, Katsushika-ku, Tokyo, 125-8585, Japan, \\ e-mail: shuji.ando@rs.tus.ac.jp
}

\section{SUMMARY}

Studies have been carried out on decomposing a model with symmetric structure using a model with asymmetric structure. In the existing decomposition theorem, the sum-symmetry model holds if and only if all of the two-parameters sum-symmetry, global symmetry and concordancediscordance models hold. However, this existing decomposition theorem does not satisfy the asymptotic equivalence for the test statistic, namely that the value of the likelihood ratio chi-squared statistic of the sumsymmetry model is asymptotically equivalent to the sum of those of the decomposed models. To address this issue, this study introduces a new decomposition theorem in which the sum-symmetry model holds if and only if all of the two-parameters sum-symmetry, global symmetry and weighted global-sum-symmetry models hold. The proposed decomposition theorem satisfies the asymptotic equivalence for the test statistic-the value of the likelihood ratio chi-squared statistic of the sum-symmetry model is asymptotically equivalent to the sum of those of the two-parameters sumsymmetry, global symmetry and weighted global-sum-symmetry models.

Key words: asymptotic equivalence, asymmetry, orthogonality, symmetry, test statistic

\section{Introduction}

Square contingency tables with the same row and column ordinal classifications are obtained by means of cross-classified matched pairs of ordinal categorical variables. In the analysis of square contingency tables, a model with symmetric structure or a model with asymmetric structure is used. 
This is because the null association (independence) model does not generally hold for square contingency tables.

The symmetry model (Bowker, 1948) is the origin of a model with symmetric structure for square contingency tables. Since then, various models with symmetric or asymmetric structure have been considered. For example, as models with symmetric structure, studies have proposed the quasisymmetry model (Caussinus, 1965), the marginal homogeneity model (Stuart, 1955), and the sum-symmetry model (Yamamoto et al., 2013); as models with asymmetric structure, studies have proposed the conditional symmetry model (McCullagh, 1978), the linear diagonals-parameter symmetry model (Agresti, 1983), the two-parameters symmetry model (Tomizawa, 1987), the conditional sum-symmetry model (Yamamoto et al., 2013), the exponential sum-symmetry model (Yamamoto et al., 2016), and the two-parameters sum-symmetry model (Yamamoto et al., 2016).

This study focuses on decomposing a model with symmetric structure using a model with asymmetric structure. The decomposition of a model is useful for searching for the reason why the model does not fit the data. Concerning the decomposition of a model with symmetric structure using a model with asymmetric structure, Read (1977) gave a decomposition theorem - the symmetry model holds if and only if both the conditional symmetry and global symmetry models hold - and showed the asymptotic equivalence for the test statistic: the value of the likelihood ratio chi-squared statistic for the symmetry model is equal to the sum of those for the conditional symmetry and global symmetry models. Using this decomposition theorem, we can see whether the fact that the symmetry model does not hold is caused by the conditional symmetry model or the global symmetry model.

Yamamoto et al. (2013) gave a decomposition theorem-the sum-symmetry model holds if and only if both the conditional sum-symmetry and global symmetry models hold - and showed the asymptotic equivalence for the test statistic: the value of the likelihood ratio chi-squared statistic for the sum-symmetry model is equal to the sum of those for the conditional sum-symmetry and global symmetry models.

Yamamoto et al. (2016) gave a decomposition theorem - the sum-symmetry model holds if and only if both the exponential sum-symmetry and global symmetry models hold. Ando (2021) pointed out that this decomposition does not satisfy the asymptotic equivalence for the test statistic. To address this issue, Ando (2021) gave an alternative decomposition the- 
orem satisfying the asymptotic equivalence for the test statistic - the sumsymmetry model holds if and only if both the exponential sum-symmetry and weighted global sum-symmetry models hold.

Moreover, Yamamoto et al. (2016) gave a decomposition theorem - the sum-symmetry model holds if and only if all of the two-parameters sumsymmetry, global symmetry and concordance-discordance models hold. We note that this decomposition also fails to satisfy the asymptotic equivalence for the test statistic. To address this issue, this study provides an alternative decomposition theorem satisfying the asymptotic equivalence for the test statistic - the sum-symmetry model holds if and only if all of the two-parameters sum-symmetry, global symmetry and weighted global sum-symmetry models hold.

The rest of this paper is organized as follows. Section 2 provides the alternative decomposition theorem satisfying the asymptotic equivalence for the test statistic. Section 3 demonstrates the utility of the proposed decomposition theorem by applying it to real data. We close with concluding remarks in section 4 .

\section{Orthogonal decomposition of the sum-symmetry model}

Consider $R \times R$ square contingency tables. Let $\pi_{i j}$ denote the probability that an observation will fall in the $(i, j)$ th cell of the table for $i=$ $1, \ldots, R ; j=1, \ldots, R$. Bowker (1948) proposed the symmetry model defined by

$$
\pi_{i j}=\pi_{j i} \quad \text { for } i \neq j .
$$

This model indicates the symmetric structure of the cell probabilities with respect to the main-diagonal cells of the table. The quasi-symmetry model (Caussinus, 1965) and the marginal homogeneity model (Stuart, 1955) indicate the symmetric structure of the odds ratios and marginal probabilities, respectively.

As a model with an asymmetric structure of the cell probabilities with respect to the main-diagonal cells of the table, we consider the following formula:

$$
\pi_{i j}=\delta_{i j} \pi_{j i} \text { for } i<j .
$$

This formula includes some models, for example: (i) the model with $\delta_{i j}=1$, for all $i<j$, is identical to the symmetry model, (ii) the model with $\delta_{i j}=\gamma$, 
for all $i<j$, is identical to the conditional symmetry model (McCullagh, 1978), (iii) the model with $\delta_{i j}=\delta^{j-i}$, for all $i<j$, is identical to the linear diagonals-parameter symmetry model (Agresti, 1983).

Tomizawa (1987) considered the two-parameters symmetry model defined by

$$
\pi_{i j}=\gamma \delta^{j-i} \pi_{j i} \text { for } i<j .
$$

This model has the following characteristics: (i) this model with $\gamma=1$ and $\delta^{j-i}=1$, for all $i<j$, is identical to the symmetry model, (ii) this model with $\delta^{j-i}=1$, for all $i<j$, is identical to the conditional symmetry model, and (iii) this model with $\gamma=1$ is identical to the linear diagonals-parameter symmetry model.

Let

$$
A_{t}=\sum_{(i, j) \in a_{t}} \sum_{i j} \quad \text { and } \quad B_{t}=\sum_{(i, j) \in b_{t}} \sum_{i j} \quad \text { for } t=3,4, \ldots, 2 R-1,
$$

where

$$
a_{t}=\{(i, j) \mid i+j=t, i<j\} \quad \text { and } \quad b_{t}=\{(i, j) \mid i+j=t, i>j\} .
$$

Yamamoto et al. (2013) proposed the sum-symmetry and conditional sum-symmetry models defined by

$$
A_{t}=B_{t} \quad \text { and } \quad A_{t}=\Gamma B_{t} \quad \text { for } t=3,4, \ldots, 2 R-1,
$$

respectively. Yamamoto et al. (2016) proposed the exponential sum-symmetry and two-parameters sum-symmetry models defined by

$$
A_{t}=\Delta^{t-2} B_{t} \quad \text { and } \quad A_{t}=\Gamma \Delta^{t-2} B_{t} \quad \text { for } t=3,4, \ldots, 2 R-1 \text {, }
$$

respectively. The two-parameters sum-symmetry model has the following characteristics: (i) the model with $\Gamma=1$ and $\Delta^{t-2}=1$, for all $t=$ $3,4, \ldots, 2 R-1$, is identical to the sum-symmetry model, (ii) the model with $\Delta^{t-2}=1$, for all $t=3,4, \ldots, 2 R-1$, is identical to the conditional sum-symmetry model, and (iii) the model with $\Gamma=1$ is identical to the exponential sum-symmetry model.

The global symmetry and concordance-discordance models are defined by

$$
\sum_{t=3}^{2 R-1} A_{t}=\sum_{t=3}^{2 R-1} B_{t} \quad \text { and } 2 \sum_{s=3}^{2 R-2} \sum_{t=s+1}^{2 R-1} A_{s} B_{t}=2 \sum_{s=3}^{2 R-2} \sum_{t=s+1}^{2 R-1} B_{s} A_{t}
$$


respectively. Yamamoto et al. (2016) gave the decomposition theorem - the sum-symmetry model holds if and only if all of the two-parameters sumsymmetry, global symmetry and concordance-discordance models hold; however, this decomposition does not satisfy the asymptotic equivalence for the test statistic. To address this issue, we propose a new decomposition theorem of the sum-symmetry model using the two-parameters sum-symmetry model. Ando (2021) introduced the weighted global sum-symmetry model defined by

$$
\sum_{t=3}^{2 R-1}(t-2) A_{t}=\sum_{t=3}^{2 R-1}(t-2) B_{t} .
$$

We obtain the following lemma.

Lemma 2.1. The sum-symmetry model holds if and only if all of the two-parameters sum-symmetry, global symmetry and weighted global sumsymmetry models hold.

Proof. The necessary condition is clear: If the sum-symmetry model holds, then all of the two-parameters sum-symmetry, global symmetry, and weighted global sum-symmetry models hold. We need to establish that the sufficient condition also holds: If all of the two-parameters sum-symmetry, global symmetry, and weighted global sum-symmetry models hold, then the sumsymmetry model holds.

Since both the two-parameters sum-symmetry and global symmetry models hold, we obtain the following equality:

$$
\Gamma=\frac{\sum_{t=3}^{2 R-1} B_{t}}{\sum_{t=3}^{2 R-1} \Delta^{t-2} B_{t}} .
$$

On the other hand, since both the two-parameters sum-symmetry and weighted global sum-symmetry models hold, we obtain the following equality:

$$
\Gamma=\frac{\sum_{t=3}^{2 R-1}(t-2) B_{t}}{\sum_{t=3}^{2 R-1}(t-2) \Delta^{t-2} B_{t}} .
$$


From the equalities (1) and (2), we obtain the following equality:

$$
\begin{aligned}
& \left(\sum_{s=3}^{2 R-1} B_{s}\right)\left(\sum_{t=3}^{2 R-1}(t-2) \Delta^{t-2} B_{t}\right)=\left(\sum_{t=3}^{2 R-1} \Delta^{t-2} B_{t}\right)\left(\sum_{s=3}^{2 R-1}(s-2) B_{s}\right) \\
& \Longleftrightarrow \sum_{t=3}^{2 R-1}\left[\left(\sum_{s=3}^{2 R-1}(t-s) B_{s}\right) \Delta^{t-2} B_{t}\right]=0 \\
& \Longleftrightarrow \sum_{t=3}^{2 R-1} \Delta^{t-2} B_{t} C_{t}=0
\end{aligned}
$$

It must be noted that $C_{t}=\sum_{s=3}^{2 R-1}(t-s) B_{s}$. Since $\sum_{s=3}^{2 R-1} \sum_{t=3}^{2 R-1}(t-$ $s) B_{s} B_{t}=0$, the equality (3) is also expressed as follows:

$$
(\Delta-1)\left[\sum_{t=4}^{2 R-1}\left(B_{2 R-1} C_{2 R-1}+\cdots+B_{t} C_{t}\right) \Delta^{t-3}\right]=0 .
$$

For $t=4, \ldots, 2 R-1$,

$$
\begin{array}{r}
B_{2 R-1} C_{2 R-1}+\cdots+B_{t} C_{t}=\sum_{k=t}^{2 R-1} \sum_{s=3}^{2 R-1}(k-s) B_{k} B_{s} \\
=\sum_{k=t}^{2 R-1} \sum_{s=3}^{t-1}(k-s) B_{k} B_{s}+\sum_{k=t}^{2 R-1} \sum_{s=t}^{2 R-1}(k-s) B_{k} B_{s} .
\end{array}
$$

The first term on the right-hand side of the equality (4) is positive, and the second term is zero. Therefore, we obtain $\Delta=1$. Additionally, from the equalities (1) and (2), we obtain $\Gamma=1$. From aforementioned result, we obtain $\Gamma=1$ and $\Delta=1$ (i.e., $A_{t}=B_{t}$ for $t=3, \ldots, 2 R-1$ ). The proof is complete.

The numbers of degrees of freedom for testing the goodness-of-fit of the sum-symmetry, conditional sum-symmetry, exponential sum-symmetry, two-parameters sum-symmetry, global symmetry, and weighted global sumsymmetry models are $2 R-3,2 R-4,2 R-4,2 R-5,1$, and 1 , respectively. It must be noted that the number of degrees of freedom for the sum-symmetry model is equal to the sum of those for the two-parameters sum-symmetry, global symmetry and weighted sum-symmetry models.

Each $f_{i j}$ denotes the observed frequency in the $(i, j)$ cell of the table $(i=1, \ldots, R ; j=1, \ldots, R)$, with a sample size $N\left(=\sum \sum f_{i j}\right)$. We assume multinomial sampling over the cells of the square contingency table. In other 
words, the observed frequencies $\left\{f_{i j}\right\}$ have a multinomial distribution with parameters that are cell probabilities $\left\{\pi_{i j}\right\}$.

Each model can be tested for goodness-of-fit by means of, for example, the likelihood ratio chi-squared statistic with the corresponding degrees of freedom. The likelihood ratio chi-squared statistic of the model $M$ is given by

$$
G^{2}(\mathrm{M})=2 \sum_{i=1}^{R} \sum_{j=1}^{R} f_{i j} \log \left(\frac{f_{i j}}{\hat{e}_{i j}}\right)
$$

where $\hat{e}_{i j}$ is the maximum likelihood estimate of the expected frequency $e_{i j}$ under the model $\mathrm{M}$.

Assume that the model $\mathrm{M}_{1}$ holds if and only if both the models $\mathrm{M}_{2}$ and $\mathrm{M}_{3}$ hold and the following asymptotic equivalence holds:

$$
G^{2}\left(\mathrm{M}_{1}\right) \simeq G^{2}\left(\mathrm{M}_{2}\right)+G^{2}\left(\mathrm{M}_{3}\right),
$$

where the number of degrees of freedom for the model $\mathrm{M}_{1}$ is equal to the sum of those for the models $\mathrm{M}_{2}$ and $\mathrm{M}_{3}$. Darroch and Silvey (1963) stated that (i) when the expression (5) holds, if both the models $\mathrm{M}_{2}$ and $\mathrm{M}_{3}$ are accepted (at the $\alpha$ significance level) with high probability, then the model $\mathrm{M}_{1}$ would be accepted. However, (ii) when the expression (5) does not hold, it will most likely give rise to an incompatible situation wherein both the models $\mathrm{M}_{2}$ and $\mathrm{M}_{3}$ are accepted with high probability, but the $\mathrm{M}_{1}$ model is rejected with high probability. This has been exemplified by Darroch and Silvey (1963) and Tahata et al. (2011). Therefore, it is preferable that the decomposition theorem satisfy the expression (5). We shall refer to the decomposition of a model satisfying the expression (5) as orthogonal decomposition.

We need to consider modifying Lemma 2.1; this is because Lemma 2.1 does not satisfy the expression (5). We consider a model that simultaneously satisfies both the global symmetry and weighted global sum-symmetry models, defined by

$$
\sum_{t=3}^{2 R-1} A_{t}=\sum_{t=3}^{2 R-1} B_{t} \quad \text { and } \quad \sum_{t=3}^{2 R-1}(t-2) A_{t}=\sum_{t=3}^{2 R-1}(t-2) B_{t} .
$$

We shall refer to this model as the double global symmetry model. The number of degrees of freedom for testing the goodness-of-fit of the double global sum-symmetry model is 2 . From Lemma 2.1, we obtain the following corollary. 
Corollary 2.1. The sum-symmetry model holds if and only if both the twoparameters sum-symmetry and double global symmetry models hold.

We obtain the following theorem.

Theorem 2.1. The following asymptotic equivalence holds:

$$
G^{2}(\mathrm{SS}) \simeq G^{2}(\mathrm{TSS})+G^{2}(\mathrm{DGS}),
$$

where $G^{2}(\mathrm{SS}), G^{2}(\mathrm{TSS})$ and $G^{2}(\mathrm{DGS})$ are the likelihood ratio chi-squared statistics of the sum-symmetry, two-parameters sum-symmetry and double global symmetry models, respectively.

Proof. Let $\boldsymbol{\pi}=\left(\pi_{11}, \pi_{12}, \ldots, \pi_{1 R}, \ldots, \pi_{R 1}, \pi_{R 2}, \ldots, \pi_{R R}\right)^{T}$, and let $\boldsymbol{A}^{T}$ denote the transpose of the matrix (or vector) $\boldsymbol{A}$. Under the two-parameters sum-symmetry model,

$$
\Gamma=\left(\frac{A_{3}}{B_{3}}\right)^{2} \frac{B_{4}}{A_{4}} \quad \text { and } \quad \Delta=\frac{B_{3} A_{4}}{A_{3} B_{4}} .
$$

Therefore, the two-parameters sum-symmetry model is expressed as follows:

$$
\boldsymbol{h}_{1}(\boldsymbol{\pi})=\mathbf{0}_{2 R-5},
$$

where

$$
\boldsymbol{h}_{1}(\boldsymbol{\pi})=\left(h_{1,5}(\boldsymbol{\pi}), h_{1,6}(\boldsymbol{\pi}), \ldots, h_{1,2 R-1}(\boldsymbol{\pi})\right)^{T}
$$

with

$$
h_{1, t}(\boldsymbol{\pi})=\left(A_{3}\right)^{t-4}\left(B_{4}\right)^{t-3} A_{t}-\left(B_{3}\right)^{t-4}\left(A_{4}\right)^{t-3} B_{t},
$$

and $\mathbf{0}_{d}$ is an $d \times 1$ vector, with all components being zero.

The double global symmetry model is expressed as

$$
\boldsymbol{h}_{2}(\boldsymbol{\pi})=\mathbf{0}_{2},
$$

where

$$
\boldsymbol{h}_{2}(\boldsymbol{\pi})=\left(h_{2,1}(\boldsymbol{\pi}), h_{2,2}(\boldsymbol{\pi})\right)^{T}
$$

with

$$
\begin{aligned}
h_{2,1}(\boldsymbol{\pi}) & =\sum_{t=3}^{2 R-1} A_{t}-\sum_{t=3}^{2 R-1} B_{t} \\
h_{2,2}(\boldsymbol{\pi}) & =\sum_{t=3}^{2 R-1}(t-2) A_{t}-\sum_{t=3}^{2 R-1}(t-2) B_{t} .
\end{aligned}
$$


From Corollary 2.1, the sum-symmetry model is expressed as

$$
\boldsymbol{h}_{3}(\boldsymbol{\pi})=\left(\boldsymbol{h}_{1}(\boldsymbol{\pi})^{T}, \boldsymbol{h}_{2}(\boldsymbol{\pi})^{T}\right)^{T}=\mathbf{0}_{2 R-3} .
$$

Let $\boldsymbol{H}_{s}(\boldsymbol{\pi})(s=1,2,3)$ denote the matrix of partial derivatives of $\boldsymbol{h}_{s}(\boldsymbol{\pi})$ with respect to $\boldsymbol{\pi}$ (i.e., $\boldsymbol{H}_{s}(\boldsymbol{\pi})=\partial \boldsymbol{h}_{s}(\boldsymbol{\pi}) / \partial \boldsymbol{\pi}^{T}$ ). Let $\boldsymbol{\Sigma}(\boldsymbol{\pi})$ be $\operatorname{diag}(\boldsymbol{\pi})-\boldsymbol{\pi} \boldsymbol{\pi}^{T}$, where $\operatorname{diag}(\boldsymbol{\pi})$ is a diagonal matrix, with the $i$ th component of $\boldsymbol{\pi}$ as the $i$ th diagonal element. We denote $\boldsymbol{p}$ as $\boldsymbol{\pi}$, with $\left\{\pi_{i j}\right\}$ replaced by $\left\{p_{i j}\right\}$, where $p_{i j}=f_{i j} / N$. Using the delta method, $\sqrt{N}\left(\boldsymbol{h}_{3}(\boldsymbol{p})-\boldsymbol{h}_{3}(\boldsymbol{\pi})\right)$ has an asymptotically normal distribution, with mean $\mathbf{0}_{2 R-3}$ and covariance matrix

$$
\boldsymbol{H}_{3}(\boldsymbol{\pi}) \boldsymbol{\Sigma}(\boldsymbol{\pi}) \boldsymbol{H}_{3}(\boldsymbol{\pi})^{T}=\left[\begin{array}{ll}
\boldsymbol{H}_{1}(\boldsymbol{\pi}) \boldsymbol{\Sigma}(\boldsymbol{\pi}) \boldsymbol{H}_{1}(\boldsymbol{\pi})^{T} & \boldsymbol{H}_{1}(\boldsymbol{\pi}) \boldsymbol{\Sigma}(\boldsymbol{\pi}) \boldsymbol{H}_{2}(\boldsymbol{\pi})^{T} \\
\boldsymbol{H}_{2}(\boldsymbol{\pi}) \boldsymbol{\Sigma}(\boldsymbol{\pi}) \boldsymbol{H}_{1}(\boldsymbol{\pi})^{T} & \boldsymbol{H}_{2}(\boldsymbol{\pi}) \boldsymbol{\Sigma}(\boldsymbol{\pi}) \boldsymbol{H}_{2}(\boldsymbol{\pi})^{T}
\end{array}\right] .
$$

All the elements of $\boldsymbol{H}_{1}(\boldsymbol{\pi}) \boldsymbol{\Sigma}(\boldsymbol{\pi}) \boldsymbol{H}_{2}(\boldsymbol{\pi})^{T}$ are equal to 0 , under the sumsymmetry model. This is because we obtain the following equalities:

$$
\begin{aligned}
& \frac{\partial h_{1, t}(\boldsymbol{\pi})}{\partial \boldsymbol{\pi}^{T}} \operatorname{diag}(\boldsymbol{\pi}) \frac{\partial h_{2,1}(\boldsymbol{\pi})}{\partial \boldsymbol{\pi}}=0 \quad \text { for } t=5, \ldots, 2 R-1, \\
& \frac{\partial h_{1, t}(\boldsymbol{\pi})}{\partial \boldsymbol{\pi}^{T}} \operatorname{diag}(\boldsymbol{\pi}) \frac{\partial h_{2,2}(\boldsymbol{\pi})}{\partial \boldsymbol{\pi}}=0 \quad \text { for } t=5, \ldots, 2 R-1, \\
& \boldsymbol{\pi}^{T} \frac{\partial h_{2,1}(\boldsymbol{\pi})}{\partial \boldsymbol{\pi}}=\sum_{t=3}^{2 R-1} A_{t}-\sum_{t=3}^{2 R-1} B_{t}, \\
& \boldsymbol{\pi}^{T} \frac{\partial h_{2,2}(\boldsymbol{\pi})}{\partial \boldsymbol{\pi}}=\sum_{t=3}^{2 R-1}(t-2) A_{t}-\sum_{t=3}^{2 R-1}(t-2) B_{t} .
\end{aligned}
$$

Therefore, we obtain

$$
\begin{aligned}
\boldsymbol{h}_{3}(\boldsymbol{\pi})^{T}\left[\boldsymbol{H}_{3}(\boldsymbol{\pi}) \boldsymbol{\Sigma}(\boldsymbol{\pi}) \boldsymbol{H}_{3}(\boldsymbol{\pi})^{T}\right]^{-1} \boldsymbol{h}_{3}(\boldsymbol{\pi}) \\
=\boldsymbol{h}_{1}(\boldsymbol{\pi})^{T}\left[\boldsymbol{H}_{1}(\boldsymbol{\pi}) \boldsymbol{\Sigma}(\boldsymbol{\pi}) \boldsymbol{H}_{1}(\boldsymbol{\pi})^{T}\right]^{-1} \boldsymbol{h}_{1}(\boldsymbol{\pi}) \\
\quad+\boldsymbol{h}_{2}(\boldsymbol{\pi})^{T}\left[\boldsymbol{H}_{2}(\boldsymbol{\pi}) \boldsymbol{\Sigma}(\boldsymbol{\pi}) \boldsymbol{H}_{2}(\boldsymbol{\pi})^{T}\right]^{-1} \boldsymbol{h}_{2}(\boldsymbol{\pi}) .
\end{aligned}
$$

Since the Wald statistic is asymptotically equivalent to the likelihood ratio statistic - see, for example, Rao (1973, Sec. 6e. 3), Darroch and Silvey (1963), and Aitchison (1962)—we obtain Theorem 2.1. The proof is complete. 
From Theorem 2.1, we see that while the Corollary 2.1 satisfies the expression (5), the decomposition theorem of Yamamoto et al. (2016) does not satisfy the expression (5). Thus, the decomposition of Theorem 2.1 is an orthogonal decomposition.

We consider a model that simultaneously satisfies both the global symmetry and concordance-discordance models, defined by

$$
\sum_{t=3}^{2 R-1} A_{t}=\sum_{t=3}^{2 R-1} B_{t} \quad \text { and } 2 \sum_{s=3}^{2 R-2} \sum_{t=s+1}^{2 R-1} A_{s} B_{t}=2 \sum_{s=3}^{2 R-2} \sum_{t=s+1}^{2 R-1} B_{s} A_{t} .
$$

We shall refer to this model as the GSCD model. In the decomposition theorem of Yamamoto et al. (2016), even if we replace both the global symmetry and concordance-discordance models with the GSCD model, it still does not satisfy the expression (5).

\section{Application to data}

We consider the dataset in Table 1, taken from Tomizawa (1985). In Table 2 , we denote the sum-symmetry, two-parameters sum-symmetry, global symmetry, weighted global sum-symmetry, double global symmetry and concordance-discordance models as the SS, TPSS, GS, WGSS, DGS and CD models, respectively. Table 2 gives the values of $G^{2}$, for each of the sum-symmetry, two-parameters sum-symmetry, global symmetry, weighted global sum-symmetry, double global symmetry, concordance-discordance and GSCD models. This table shows that the two-parameters sum-symmetry and concordance-discordance models fit well, but the other models fit poorly. Under the two-parameters sum-symmetry model, the maximum likelihood estimates of the parameters $\Gamma$ and $\Delta$ are 0.894 and 0.972 , respectively. Thus, the maximum likelihood estimates of $\Gamma \Delta^{t-2}$, for all $t=3,4,5,6,7$, are less than 1 . Therefore, a university student's left eye grade is estimated to be better than his/her right eye grade. If the double global symmetry model fits well for the dataset in Table 1, then we can make the interpretation that a university student's left eye grade is estimated to be equivalent to his/her right eye grade.

We want to detect the cause of the fact that the sum-symmetry model does not fit the dataset in Table 1. From Lemma 2.1, we can infer that the sum-symmetry model does not hold for the dataset in Table 1 because of the global symmetry and weighted global sum-symmetry models rather than because of the two-parameters sum-symmetry model. From the decomposition 
Table 1. Dataset of unaided vision data of 4,746 university students in Japan; source Tomizawa (1985).

\begin{tabular}{rrrrrr}
\hline & \multicolumn{4}{c}{ Left eye grades } & \\
\cline { 2 - 5 } Right eye grades & $(1)$ & $(2)$ & $(3)$ & $(4)$ & Total \\
\hline Highest (1) & 1291 & 130 & 40 & 22 & 1483 \\
Second (2) & 149 & 221 & 114 & 23 & 507 \\
Third (3) & 64 & 124 & 660 & 185 & 1033 \\
Lowest (4) & 20 & 25 & 249 & 1429 & 1723 \\
Total & 1524 & 500 & 1063 & 1659 & 4746 \\
\hline
\end{tabular}

Table 2. Values of the likelihood ratio chi-squared statistic $\left(G^{2}\right)$ for the models applied to the data of Table 1.

\begin{tabular}{ccc}
\hline Models & Degrees of freedom & $G^{2}$ \\
\hline SS & 5 & $16.668^{*}$ \\
TSS & 3 & 4.086 \\
GS & 1 & $11.976^{*}$ \\
WGSS & 1 & $11.867^{*}$ \\
DGS & 2 & $12.584^{*}$ \\
CD & 1 & 0.648 \\
GSCD & 2 & $12.625^{*}$ \\
\hline
\end{tabular}

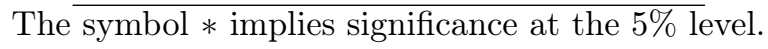

theorem of Yamamoto et al. (2016), we can infer that the sum-symmetry model does not hold because of the global symmetry model rather than because of the two-parameters sum-symmetry and concordance-discordance models.

In this context, we point out that (i) the value of the likelihood ratio chi-squared statistic of the sum-symmetry model is nearly equal to the sum of those of the two-parameters sum-symmetry and double global symmetry models, (ii) the value of the likelihood ratio chi-squared statistic of the sum-symmetry model is slightly greater than the sum of those of the twoparameters sum-symmetry and GSCD models, and (iii) the value of the likelihood ratio chi-squared statistic of the sum-symmetry model is greater than the sum of those of the two-parameters sum-symmetry, global symmetry and weighted global sum-symmetry models. 


\section{Concluding remarks}

This study has established an alternative decomposition theorem in which the sum-symmetry model holds if and only if all of the two-parameters sum-symmetry, global symmetry and weighted global sum-symmetry models hold (i.e., Lemma 2.1 or Corollary 2.1), and has shown that the value of the likelihood ratio chi-squared statistic of the sum-symmetry model is asymptotically equivalent to the sum of those of the two-parameters sumsymmetry and double global symmetry models (i.e., Theorem 2.1).

\section{Acknowledgements}

The author would like to thank the anonymous reviewers and editors for their comments and suggestions for improvements to this paper.

\section{REFERENCES}

Agresti, A. (1983): A simple diagonals-parameter symmetry and quasi-symmetry model. Statistics \& Probability Letters 1: 313-316.

Aitchison, J. (1962): Large-sample restricted parametric tests. Journal of the Royal Statistical Society: Series B 24: 234-250.

Ando, S. (2021): Orthogonal decomposition of the sum-symmetry model for square contingency tables with ordinal categories: Use of the exponential sumsymmetry model. Biometrical Letters: in press.

Bowker, A. H. (1948): A test for symmetry in contingency tables. Journal of the American Statistical Association 43: 572-574.

Caussinus, H. (1965): Contribution à l'Analyse Statistique des Tableaux de Corrélation. Annales de la Faculté des Sciences de l'Université de Toulouse 29: $77-183$.

Darroch, J.N., Silvey, S.D. (1963): On testing more than one hypothesis. The Annals of Mathematical Statistics 34: 555-567.

McCullagh, P. (1978): A class of parametric models for the analysis of square contingency tables with ordered categories. Biometrika 65: 413-418.

Rao, C.R. (1973): Linear statistical inference and its applications, 2nd ed. Wiley New York.

Read, C.B. (1977): Partitioning chi-squape in contingency tables: A teaching approach. Communications in Statistics - Theory and Methods 6: 553-562.

Stuart, A. (1955): A test for homogeneity of the marginal distributions in a twoway classification. Biometrika 42: 412-416.

Tahata, K., Ando, S., Tomizawa, S. (2011): Ridit score type asymmetry model and decomposition of symmetry for square contingency tables. Model Assisted Statistics and Applications 6: 279-286.

Tomizawa, S. (1985): Analysis of data in square contingency tables with ordered categories using the conditional symmetry model and its decomposed models. Environmental Health Perspectives 63: 235-239. 
Tomizawa, S. (1987): Decompositions for 2-ratios-parameter symmetry model in square contingency tables with ordered categories. Biometrical Journal 29: $45-55$.

Yamamoto, K., Tanaka, Y., Tomizawa, S. (2013): Sum-symmetry model and its orthogonal decomposition for square contingency tables with ordered categories. SUT Journal of Mathematics 49: 121-128.

Yamamoto, K., Aizawa, M., Tomizawa, S. (2016): Decomposition of sum-symmetry model for ordinal square contingency tables. European Journal of Statistics and Probability 4: 12-19. 